\title{
Predicting Heterogeneity of Gravel Influences on Concrete Tensile Strength Partially Replaced Cement with Metakaolin Content
}

\author{
Eluozo $\mathrm{SN}^{1 *}$ and Dimkpa $\mathrm{K}^{2}$ \\ ${ }^{1}$ Department of Civil Engineering, Gregory University Uturu (GUU), Nigeria \\ ${ }^{2}$ Department of Architecture, Rivers State University, Nigeria
}

*Corresponding author: Eluozo SN, Department of Civil Engineering, College of Engineering, Nigeria

Gregory University Uturu (GUU), Abia State of Nigeria

\begin{abstract}
Heterogeneity of aggregate in concrete has been monitored in different developed mixed design concrete by experts applying different curing techniques. Their findings have always reflected the variation of aggregate size as one of the major influential property that determined various rates of tensile, based on its compressive strength. The behaviour are basically on the effect from aggregate shapes that reflect on the generated results on the model concrete, but other parameters that express impact on the variation rate of tensile strength are not always determined, because of the limitation of experimental techniques including the concept of ignoring these other parameters on concrete properties , these are other major significant areas that will determine various rate of tensile partially replaced cement with Metakaolin substance, the study applied modeling and simulation using derived analytical solution, the developed model generated simulation values that produced various rates of tensile strength parameters at the optimum values recorded at ninety days of curing, decrease of the tensile were observed based on the reflection of aggregate heterogeneity, compaction rate mixed time and curing techniques were experienced in the study, the impact of heterogeneous aggregate on tensile strength including variation of water cement ratios were observed, the predictive and experimental values were monitored and both parameters developed best fits. The study is imperative because the application of simulation techniques has expressed the impact of various parameters that there impact were not observed in the experimental development of concrete generating tensile strength.
\end{abstract}

Keywords: Predicting; Heterogeneity; Gravel Cement and Metakaolin

\section{Introduction}

Several studies have been carried out in various dimensions, this was carried out to monitor the application of the localized materials, and these are iron slag waste tyre and Rice husk Ash as partial replacement of cement. other materials are fine and coarse aggregates that develop strength, these type of techniques are now available in the literature [1-7], the techniques has produced high required result, the method of applying waste tyre were observed and it has generated tremendous feasibility using gargantuan amounts from waste tyre on concrete products. Experts has also applied other materials such as plastic waste, this concept become a better option because it has solved some environmental issues around the globe Choi et al. [8] current studies that familiar has examined the effect of waste PET bottles as aggregate on properties of concrete. It has been definitely yielding tremendous results because this technique of applying waste tyre and bottles has reduced by $2-6 \%$ of normal weight concrete. Marzouk et al. [9], while other studies carried out on the applications of consumed plastic bottle as waste material for partial sand replacement; the study from its thorough examinations monitored that density was lowered when the PET aggregate exceeding $50 \%$ by volume of sand. Meanwhile Suganthy et al. [10] examined the reduction in weight of concrete compared to plastic content and it experienced 
an increase Ode and Eluozo [11]. Marzouk [9] other research was carried out to examine the reduction of compressive strength from plastic concrete; other experts in the same vein applied techniques of partial replacement of sand with plastic. Al-Manasser and Dalal (1997) this investigation was carried out by monitoring the influence of plastic on concrete mix. It was observed that the splitting tensile strength experienced decreased as the plastic content increased. Batayneh et al. [12] express splitting tensile and flexural strength of concrete mix slump on the replacement and observed that the plastic content an increase in both materials. Numerous researchers have also investigated the strengths of plastic concrete, Batayneh et al. [12] mentioned that the integration of ground plastic in concrete and it was observed to have an effect on its compressive strength [13-15] investigated the effect of postconsumer waste plastic in concrete as a soft filer [16-20].

\section{Theoretical Background}

$$
\begin{aligned}
& \text { Nomenclature } \\
& \mathrm{C}=\text { Tensile Strength } \\
& \mathrm{D}=\text { Cementious materials } \\
& \mathrm{K}=\text { Concrete porosity and void ratios } \\
& 2 \mathrm{n}=\text { Water Cement Ratios } \\
& \mathrm{X}=\text { Curing Age } \\
& \frac{d}{\mathbb{X}}+A_{(X)} C_{(d)}=K_{(X)} C_{d}{ }^{n} ; n \geq 2 \\
& \text { Let } \beta=C_{d}{ }^{1-n} \\
& \frac{d \beta}{\mathbb{X}}=(1-n) C_{d}{ }^{-n} \frac{d}{\mathbb{X}} \\
& C_{d}{ }^{-n} \frac{d}{\mathscr{d}}=\left(\frac{1}{1-n}\right) \frac{d \beta}{\mathbb{X}} \\
& \mathrm{But}^{\beta}=C_{d}{ }^{1-n} \\
& C_{d}{ }^{1-n}=2 D \exp \left[(2 n-2) \int K_{(x)}\right. \\
& C_{d}{ }^{1-n}=D \exp \left[(2 n-2) K_{(x)} X\right]
\end{aligned}
$$

\section{Materials and Method}

\section{Apparatus required tensile strength}

1. Weights and weighing device.

2. Tools, containers and pans for carrying materials \& mixing.

3. A circular cross-sectional rod ( $\varphi \mathrm{l} 6 \mathrm{~mm} \& 600 \mathrm{~mm}$ length).

4. $\quad$ Testing machine.5.

5. Three cylinders $(\varphi 150 \mathrm{~mm} \& 300 \mathrm{~mm}$ in height).

6. A jig for aligning concrete cylinder and bearing strips.
Prepare three cylindrical concrete specimens following same steps as test No.32:

1. After moulding and curing the specimens for seven days in water, they can be tested.

2. Two bearings strips of nominal $(1 / 8$ in i.e $3.175 \mathrm{~mm})$ thick plywood, free of imperfections, approximately $(25 \mathrm{~mm})$ wide, and of length equal to or slightly longer than that of the specimen should be provided for each specimen.

3. The bearing strips are placed between the specimen and both upper and lower bearing blocks of the testing machine or between the specimen and the supplemental bars or plates.

4. Draw diametric lines each end of the specimen using a suitable device that will ensure that they are in the same axial plane. Centre one of the plywood strips along the Centre of the lower bearing block.

5. Place the specimen on the plywood strip and align so that the lines marked on the ends of the specimen are vertical and centered over the plywood strip.

6. Place a second plywood strip lengthwise on the cylinder, centered on the lines marked on the ends of the cylinder.

7. Apply the load continuously and without shock, at a constant rate within, the range of 689 to $1380, \mathrm{kPa} / \mathrm{min}$ splitting tensile stress until failure of the specimen.

8. Record the maximum applied load indicated by the testing machine at failure. Note the type of failure and appearance of fracture.

\section{Observations and calculations}

Calculate the splitting tensile strength of the specimen as follows:

$$
\mathrm{T}=2 \mathrm{P} / \mathrm{Ld}
$$

Where: T: splitting tensile strength, $\mathrm{N} / \mathrm{mm}^{2} \mathrm{P}$ : maximum applied load indicated by testing machine, NL: Length of the specimen, mmd: diameter of the specimen, $\mathrm{mm}$

\section{Results and Discussion}

Table 1-6 and Figure 1-6.

The growth rate of the tensile strength were also linked with the behaviour the aggregate size in the various mixed proportions, the heterogeneity of the aggregates in size reflected the maximum tensile strength in different water cement ratios, based on these factors, the growth rate were affected, these are based on these influential material in the model concrete grades, this implies that the heterogeneity that reflected the surfaced area for developments of gel bond become responsible for the variations of the developed strength at various mixed proportions, these factors in the model 
concrete study implies that bigger aggregate size causes a more heterogeneity in the concrete which will prevent the uniform distribution of load when stressed. The shape and texture of aggregate affects the properties of fresh concrete more than hardened concrete. This implies that there would have been rapid in tensile strength if the aggregate shape has the require quality texture, because it affect the properties of fresh concrete more than harden concrete. Aggregate particles that are cubicle or spherical in shape with correct mineral composition are ideal for maximizing concrete strength. The trend observed the effect from these behaviour of aggregate heterogeneity based on these stated factors, these expressed the graphical representation on linear growth rate, but the results observed decrease in different mixed proportion based on the variation of aggregate size, the results even on exponential trend explained the decrease in strength that will definitely prevent uniformity distribution of load, other factors from the trend that decrease the tensile strength are reflection from the variation of different mixed proportion on the developed model concrete, while other are from the rate of degree of compaction, mixed time, different techniques in curing, including the variation size of the aggregate. The results from these presented figures were monitor based these expressed factors. The figures show the results on linear phase that observed the variation in all the designed parameters to determine the effect it has on tensile strength.

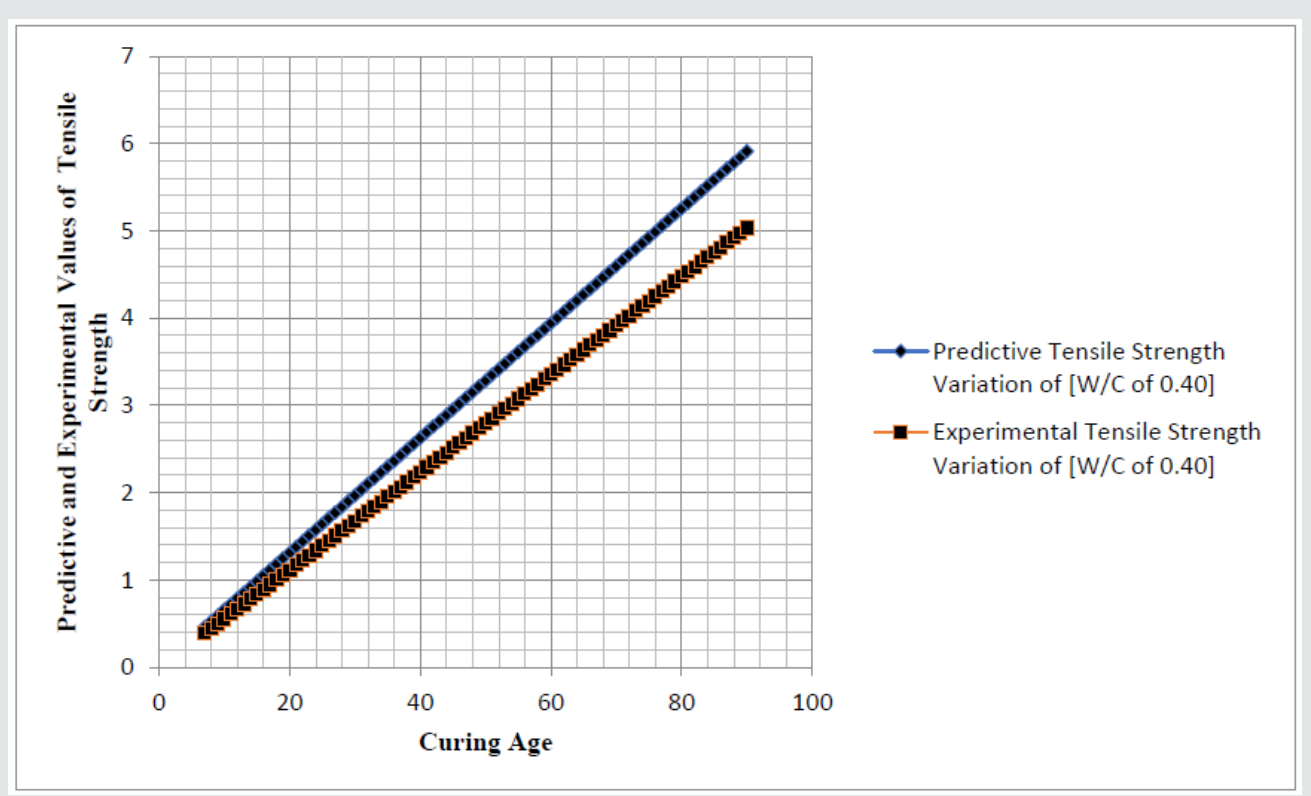

Figure 1: Predictive and Experimental Value of Split Tensile Techniques at Different Curing Age.

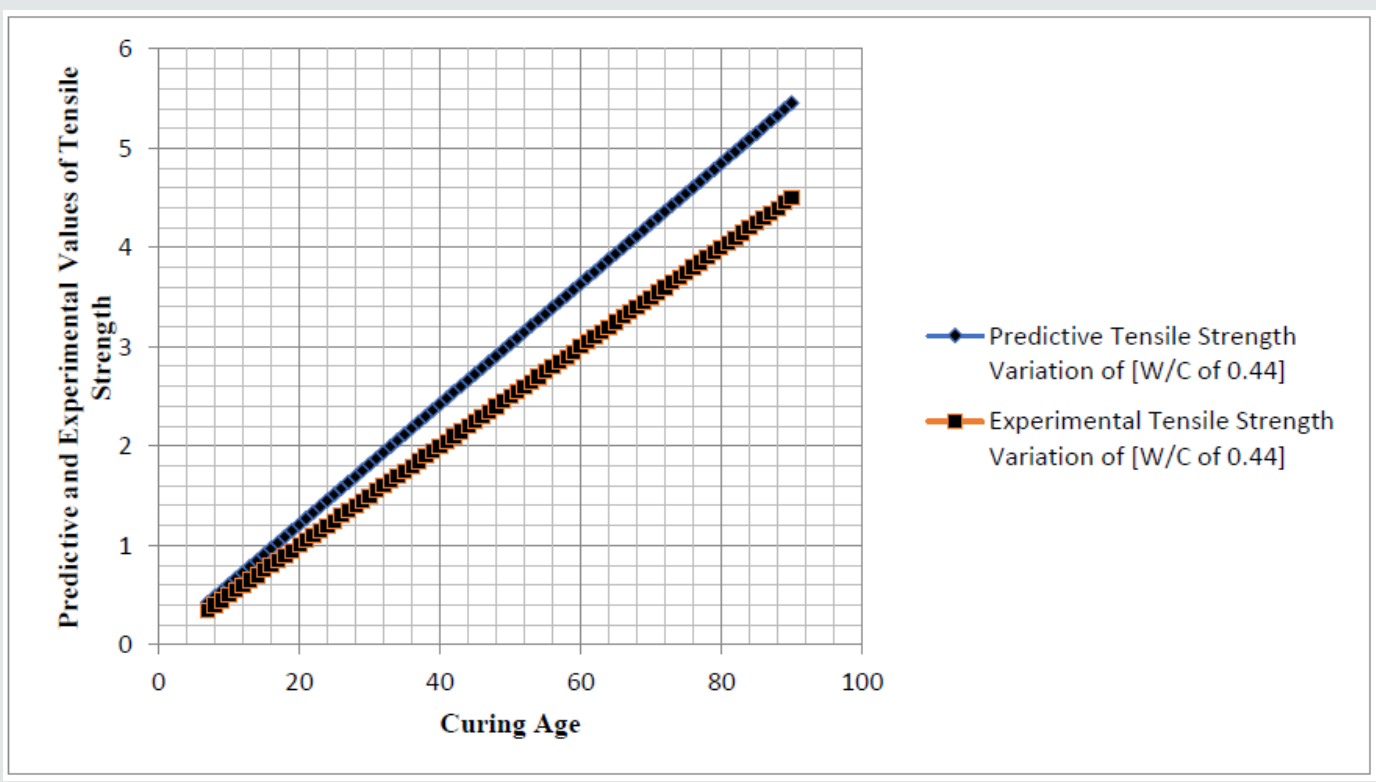

Figure 2: Predictive and Experimental Value of Split Tensile Techniques at Different Curing Age. 


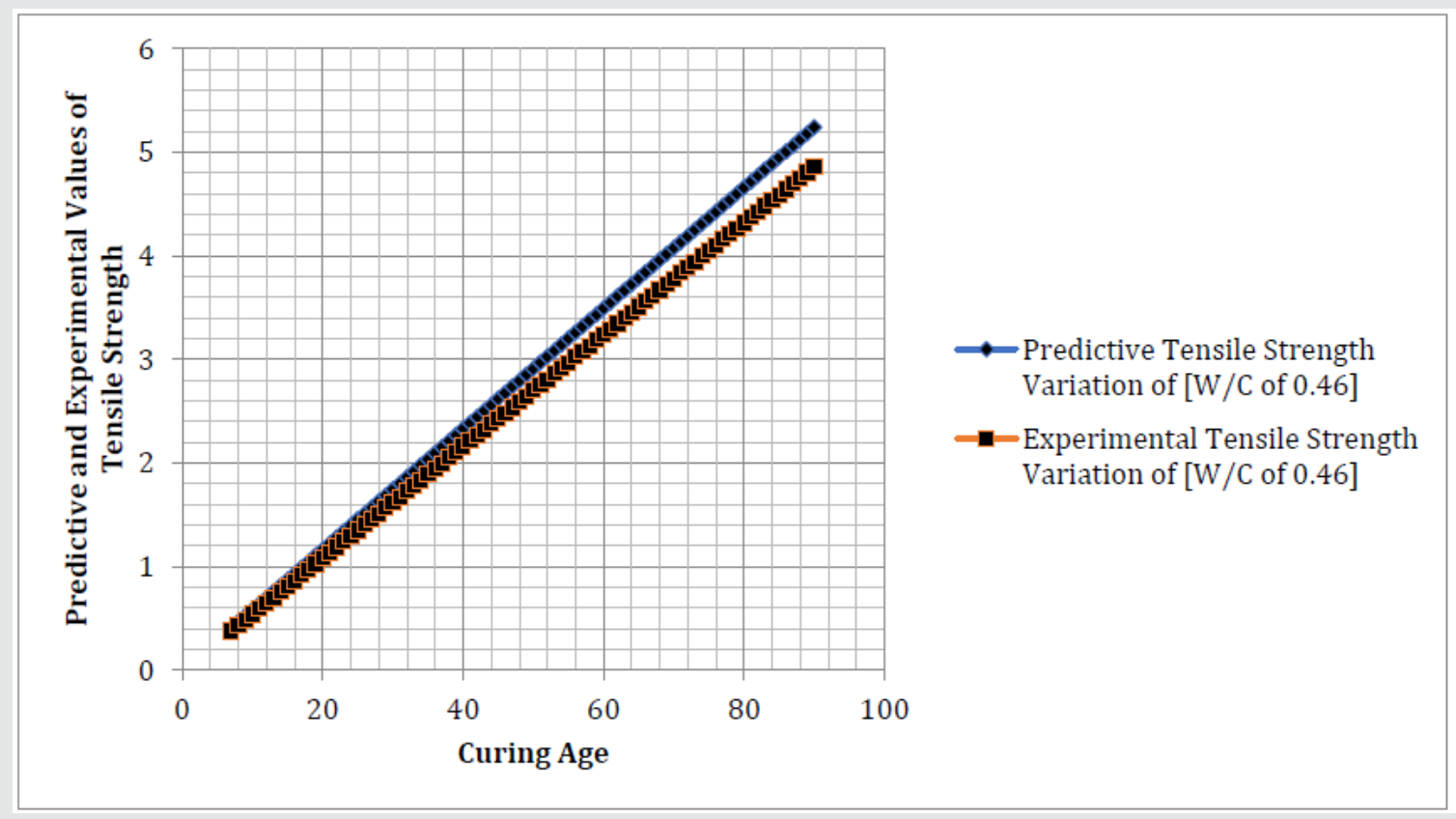

Figure 3: Predictive and Experimental Value of Split Tensile Techniques at Different Curing Age.

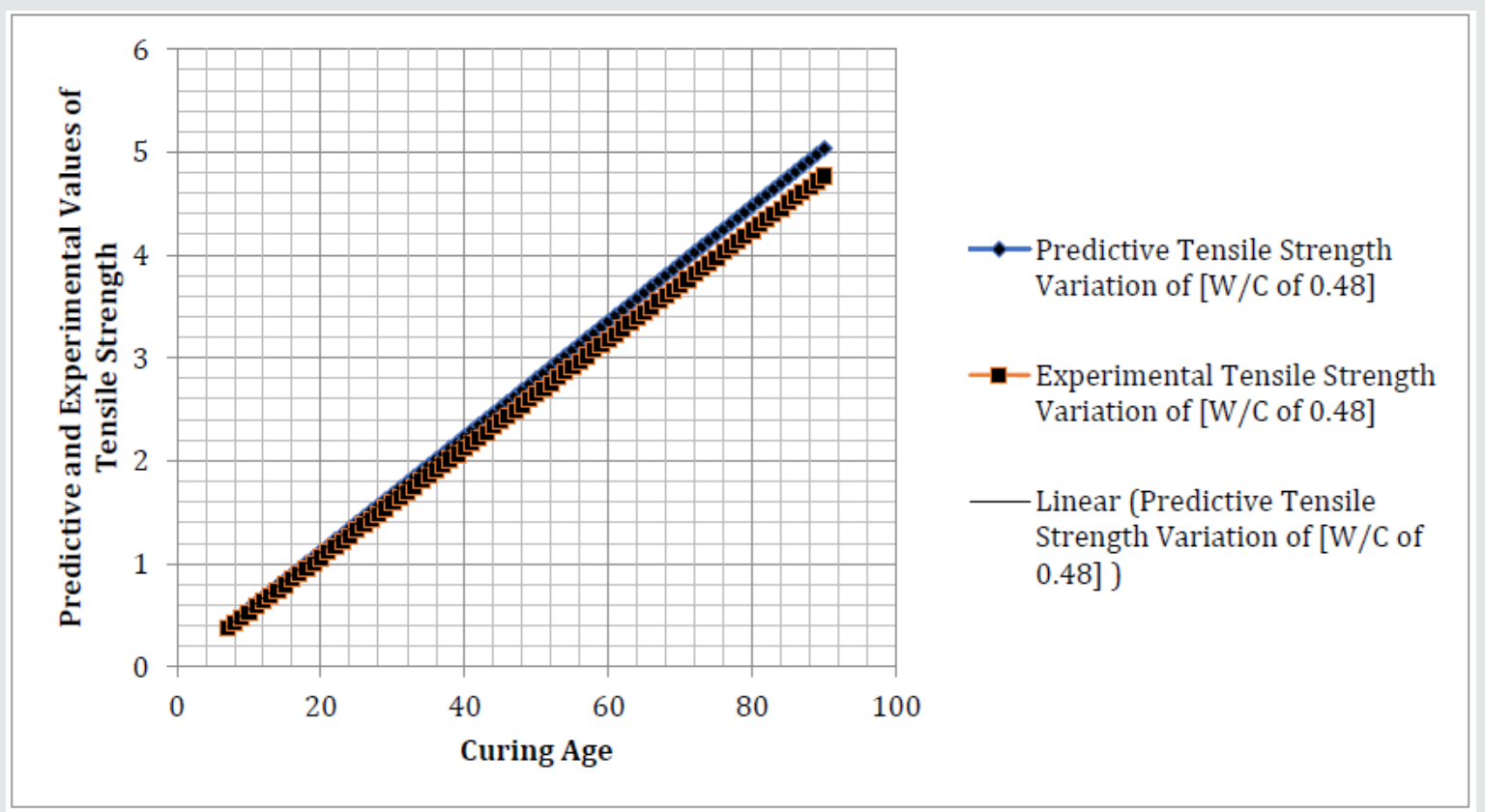

Figure 4: Predictive and Experimental Value of Split Tensile Techniques at Different Curing Age. 


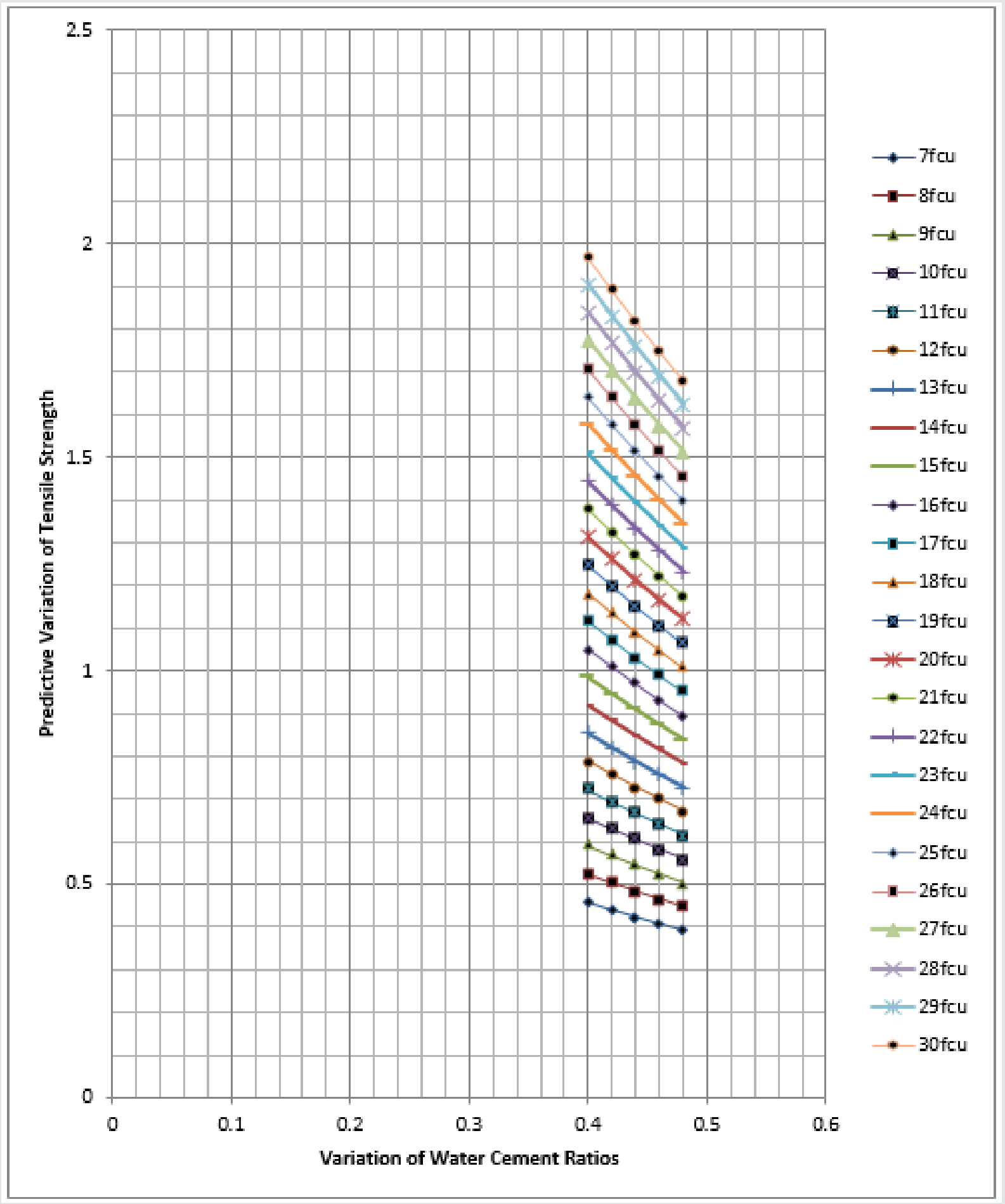

Figure 6a: Variation of Predictive Values of Split Tensile Techniques at Different Water cement Ratios Curing Age. 


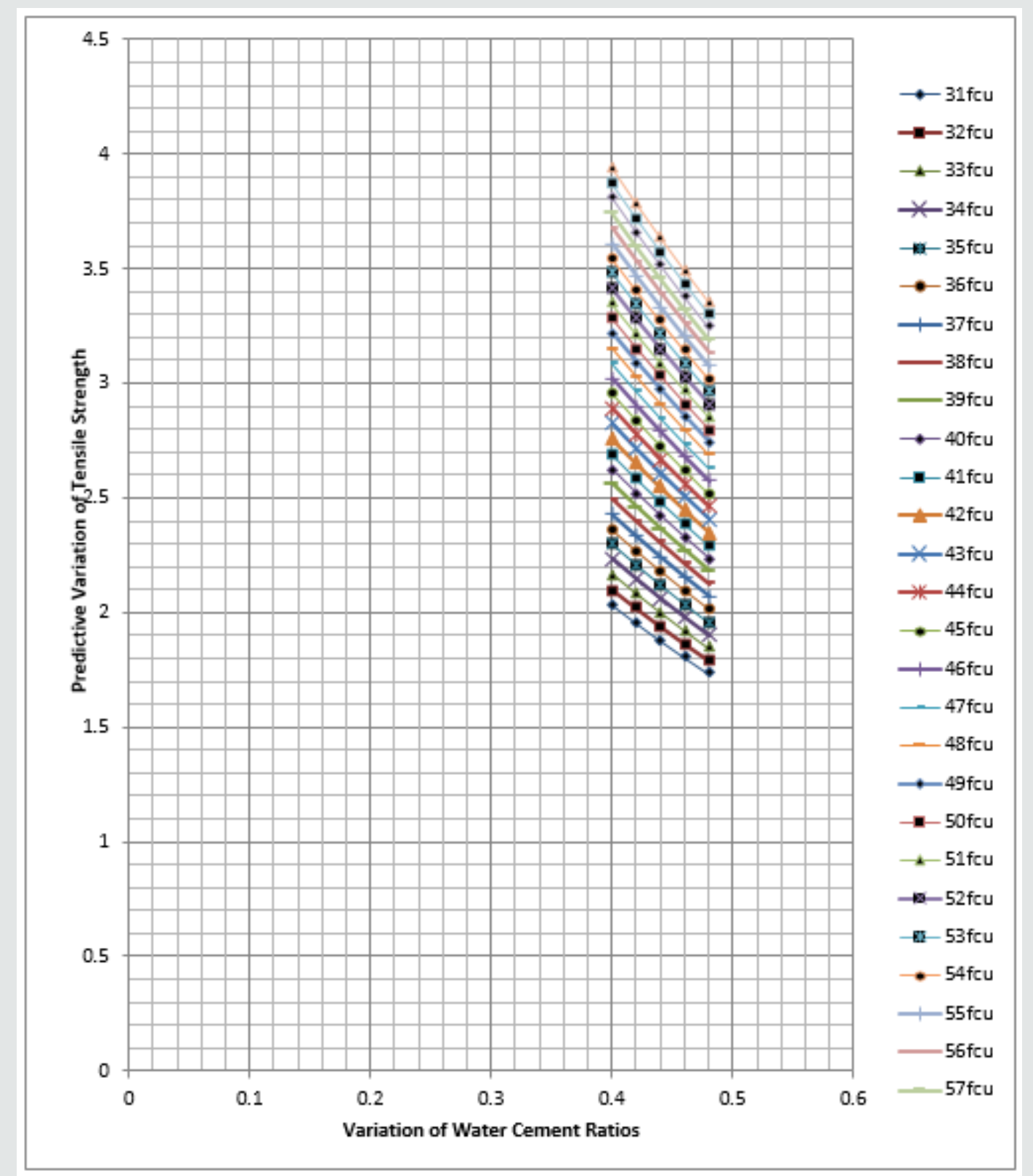

Figure 6b: Variation of Predictive Values of Split Tensile Techniques at Different Water cement Ratios Curing Age 


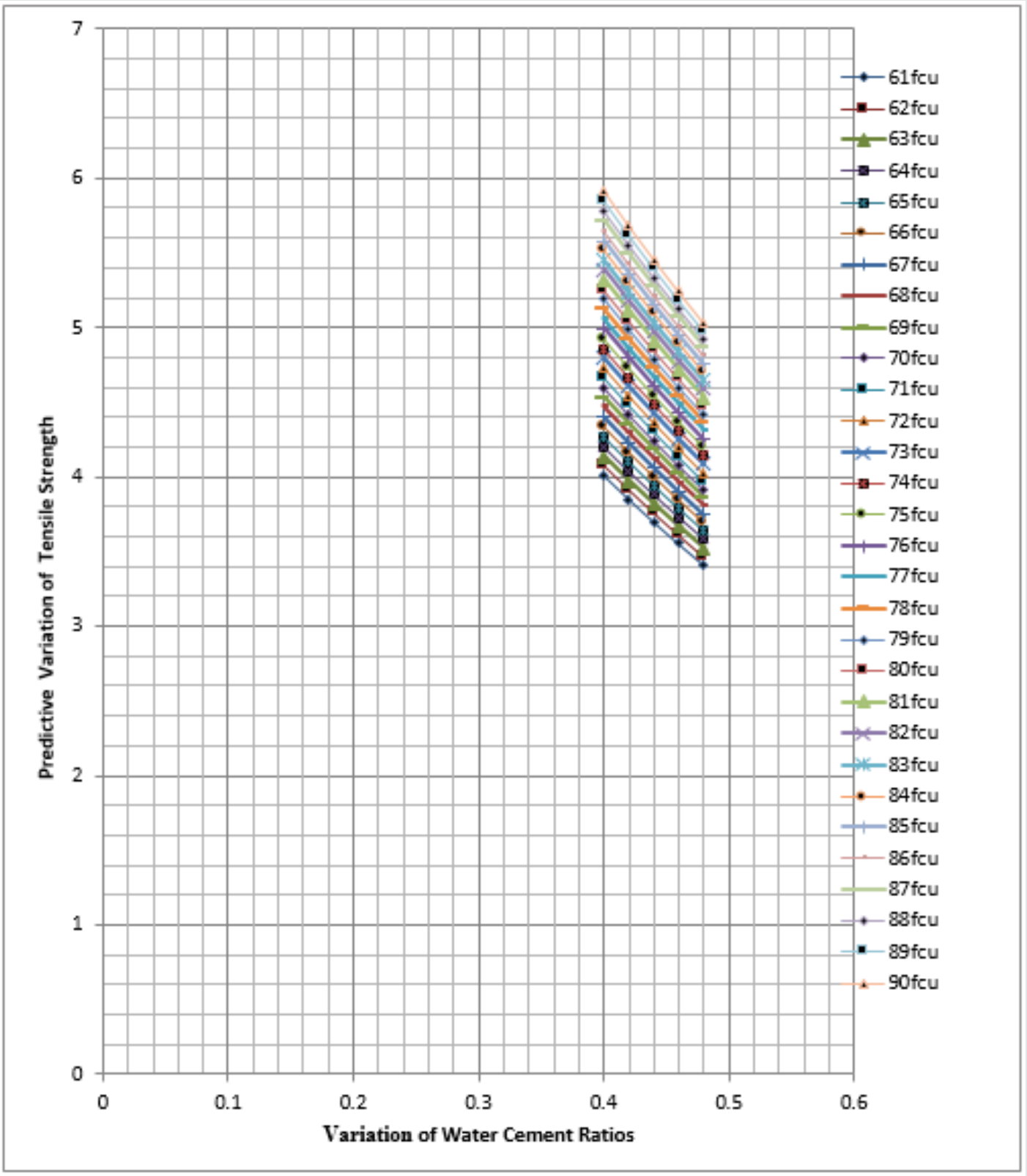

Figure 6C: Variation of Predictive Values of Split Tensile Techniques at Different Water cement Ratios Curing Age

Table 1: Predictive and Experimental Value of Split Tensile Techniques at Different Curing Age.

\begin{tabular}{|c|c|c|}
\hline Curing Age & $\begin{array}{c}\text { Predictive Tensile Strength Variation of [W/C of } \\
\mathbf{0 . 4 0 ]}\end{array}$ & $\begin{array}{c}\text { Experimental Tensile Strength Variation of } \\
\text { [W/C of 0.40] }\end{array}$ \\
\hline 7 & 0.459653 & 0.387 \\
\hline 8 & 0.525318 & 0.443 \\
\hline 9 & 0.590982 & 0.499 \\
\hline 10 & 0.656647 & 0.555 \\
\hline 11 & 0.722312 & 0.611 \\
\hline 12 & 0.787976 & 0.667 \\
\hline 13 & 0.853641 & 0.723 \\
\hline 14 & 0.919306 & 0.779 \\
\hline 15 & 0.98497 & 0.835 \\
\hline
\end{tabular}




\begin{tabular}{|c|c|c|}
\hline 16 & 1.050635 & 0.891 \\
\hline 17 & 1.1163 & 0.947 \\
\hline 18 & 1.181964 & 1.003 \\
\hline 19 & 1.247629 & 1.059 \\
\hline 20 & 1.313294 & 1.115 \\
\hline 21 & 1.378958 & 1.171 \\
\hline 22 & 1.444623 & 1.227 \\
\hline 23 & 1.510288 & 1.283 \\
\hline 24 & 1.575953 & 1.339 \\
\hline 25 & 1.641617 & 1.395 \\
\hline 26 & 1.707282 & 1.451 \\
\hline 27 & 1.772947 & 1.507 \\
\hline 28 & 1.838611 & 1.563 \\
\hline 29 & 1.904276 & 1.619 \\
\hline 30 & 1.969941 & 1.675 \\
\hline 31 & 2.035605 & 1.731 \\
\hline 32 & 2.10127 & 1.787 \\
\hline 33 & 2.166935 & 1.843 \\
\hline 34 & 2.232599 & 1.899 \\
\hline 35 & 2.298264 & 1.955 \\
\hline 36 & 2.363929 & 2.011 \\
\hline 37 & 2.429593 & 2.067 \\
\hline 38 & 2.495258 & 2.123 \\
\hline 39 & 2.560923 & 2.179 \\
\hline 40 & 2.626588 & 2.235 \\
\hline 41 & 2.692252 & 2.291 \\
\hline 42 & 2.757917 & 2.347 \\
\hline 43 & 2.823582 & 2.403 \\
\hline 44 & 2.889246 & 2.459 \\
\hline 45 & 2.954911 & 2.515 \\
\hline 46 & 3.020576 & 2.571 \\
\hline 47 & 3.08624 & 2.627 \\
\hline 48 & 3.151905 & 2.683 \\
\hline 49 & 3.21757 & 2.739 \\
\hline 50 & 3.283234 & 2.795 \\
\hline 51 & 3.348899 & 2.851 \\
\hline 52 & 3.414564 & 2.907 \\
\hline 53 & 3.480228 & 2.963 \\
\hline 54 & 3.545893 & 3.019 \\
\hline 55 & 3.611558 & 3.075 \\
\hline 56 & 3.677223 & 3.131 \\
\hline 57 & 3.742887 & 3.187 \\
\hline 58 & 3.808552 & 3.243 \\
\hline 59 & 3.874217 & 3.299 \\
\hline 60 & 3.939881 & 3.355 \\
\hline 61 & 4.005546 & 3.411 \\
\hline 62 & 4.071211 & 3.467 \\
\hline 63 & 4.136875 & 3.523 \\
\hline
\end{tabular}




\begin{tabular}{|c|c|c|}
\hline 64 & 4.20254 & 3.579 \\
\hline 65 & 4.268205 & 3.635 \\
\hline 66 & 4.333869 & 3.691 \\
\hline 67 & 4.399534 & 3.747 \\
\hline 68 & 4.465199 & 3.803 \\
\hline 69 & 4.530863 & 3.859 \\
\hline 70 & 4.596528 & 3.915 \\
\hline 71 & 4.662193 & 3.971 \\
\hline 72 & 4.727858 & 4.027 \\
\hline 73 & 4.793522 & 4.083 \\
\hline 74 & 4.859187 & 4.139 \\
\hline 75 & 4.924852 & 4.195 \\
\hline 76 & 4.990516 & 4.251 \\
\hline 77 & 5.056181 & 4.307 \\
\hline 78 & 5.121846 & 4.363 \\
\hline 79 & 5.18751 & 4.419 \\
\hline 80 & 5.253175 & 4.475 \\
\hline 81 & 5.31884 & 4.531 \\
\hline 82 & 5.384504 & 4.587 \\
\hline 83 & 5.450169 & 4.643 \\
\hline 84 & 5.515834 & 4.699 \\
\hline 85 & 5.581498 & 4.755 \\
\hline 86 & 5.647163 & 4.811 \\
\hline 87 & 5.712828 & 4.867 \\
\hline 88 & 5.778493 & 4.923 \\
\hline 89 & 5.844157 & 4.979 \\
\hline 90 & 5.909822 & 5.035 \\
\hline
\end{tabular}

Table 2: Predictive and Experimental Value of Split Tensile Techniques at Different Curing Age.

\begin{tabular}{|c|c|c|}
\hline Curing Age & $\begin{array}{c}\text { Predictive Tensile Strength Variation of [W/C of } \\
\mathbf{0 . 4 2}]\end{array}$ & $\begin{array}{c}\text { Experimental Tensile Strength Variation of } \\
\text { [W/C of 0.42] }\end{array}$ \\
\hline 7 & 0.44163 & 0.371 \\
\hline 8 & 0.50472 & 0.424 \\
\hline 9 & 0.567809 & 0.477 \\
\hline 10 & 0.630899 & 0.53 \\
\hline 11 & 0.693989 & 0.636 \\
\hline 12 & 0.757079 & 0.689 \\
\hline 13 & 0.820169 & 0.742 \\
\hline 14 & 0.883259 & 0.795 \\
\hline 15 & 0.946349 & 0.848 \\
\hline 16 & 1.009439 & 0.901 \\
\hline 17 & 1.072529 & 0.954 \\
\hline 18 & 1.135619 & 1.007 \\
\hline 19 & 1.198709 & 1.06 \\
\hline 21 & 1.261799 & 1.113 \\
\hline 22 & 1.324889 & 1.166 \\
\hline 23 & 1.387979 & 1.219 \\
\hline
\end{tabular}




\begin{tabular}{|c|c|c|}
\hline 24 & 1.514159 & 1.272 \\
\hline 25 & 1.577248 & 1.325 \\
\hline 26 & 1.640338 & 1.378 \\
\hline 27 & 1.703428 & 1.431 \\
\hline 28 & 1.766518 & 1.484 \\
\hline 29 & 1.829608 & 1.537 \\
\hline 30 & 1.892698 & 1.59 \\
\hline 31 & 1.955788 & 1.643 \\
\hline 32 & 2.018878 & 1.696 \\
\hline 33 & 2.081968 & 1.749 \\
\hline 34 & 2.145058 & 1.802 \\
\hline 35 & 2.208148 & 1.855 \\
\hline 36 & 2.271238 & 1.908 \\
\hline 37 & 2.334328 & 1.961 \\
\hline 38 & 2.397418 & 2.014 \\
\hline 39 & 2.460508 & 2.067 \\
\hline 40 & 2.523598 & 2.12 \\
\hline 41 & 2.586687 & 2.173 \\
\hline 42 & 2.649777 & 2.226 \\
\hline 43 & 2.712867 & 2.279 \\
\hline 44 & 2.775957 & 2.332 \\
\hline 45 & 2.839047 & 2.385 \\
\hline 46 & 2.902137 & 2.438 \\
\hline 47 & 2.965227 & 2.491 \\
\hline 48 & 3.028317 & 2.544 \\
\hline 49 & 3.091407 & 2.597 \\
\hline 50 & 3.154497 & 2.65 \\
\hline 51 & 3.217587 & 2.703 \\
\hline 52 & 3.280677 & 2.756 \\
\hline 53 & 3.343767 & 2.809 \\
\hline 54 & 3.406857 & 2.862 \\
\hline 55 & 3.469947 & 2.915 \\
\hline 56 & 3.533037 & 2.968 \\
\hline 57 & 3.596127 & 3.021 \\
\hline 58 & 3.659216 & 3.074 \\
\hline 59 & 3.722306 & 3.127 \\
\hline 60 & 3.785396 & 3.18 \\
\hline 61 & 3.848486 & 3.233 \\
\hline 62 & 3.911576 & 3.286 \\
\hline 63 & 3.974666 & 3.339 \\
\hline 64 & 4.037756 & 3.392 \\
\hline 65 & 4.100846 & 3.445 \\
\hline 66 & 4.163936 & 3.498 \\
\hline 67 & 4.227026 & 3.551 \\
\hline 68 & 4.290116 & 3.604 \\
\hline 69 & 4.353206 & 3.657 \\
\hline 70 & 4.416296 & 3.71 \\
\hline
\end{tabular}




\begin{tabular}{|c|c|c|}
\hline 71 & 4.479386 & 3.763 \\
\hline 72 & 4.542476 & 3.816 \\
\hline 73 & 4.605566 & 3.869 \\
\hline 74 & 4.668655 & 3.922 \\
\hline 75 & 4.731745 & 3.975 \\
\hline 76 & 4.794835 & 4.028 \\
\hline 77 & 4.857925 & 4.081 \\
\hline 78 & 4.921015 & 4.134 \\
\hline 79 & 4.984105 & 4.187 \\
\hline 80 & 5.047195 & 4.24 \\
\hline 81 & 5.110285 & 4.293 \\
\hline 82 & 5.173375 & 4.346 \\
\hline 83 & 5.236465 & 4.399 \\
\hline 84 & 5.299555 & 4.452 \\
\hline 85 & 5.362645 & 4.505 \\
\hline 86 & 5.425735 & 4.558 \\
\hline 87 & 5.488825 & 4.611 \\
\hline 88 & 5.551915 & 4.664 \\
\hline 89 & 5.615005 & 4.717 \\
\hline 90 & 5.678094 & 4.77 \\
\hline
\end{tabular}

Table 3: Predictive and Experimental Value of Split Tensile Techniques at Different Curing Age.

\begin{tabular}{|c|c|c|}
\hline Curing Age & $\begin{array}{l}\text { Predictive Tensile Strength Variation of [W/C of } \\
0.44]\end{array}$ & $\begin{array}{l}\text { Experimental Tensile Strength Variation of } \\
\text { [W/C of } 0.44]\end{array}$ \\
\hline 7 & 0.424313 & 0.35 \\
\hline 8 & 0.484929 & 0.4 \\
\hline 9 & 0.545545 & 0.45 \\
\hline 10 & 0.606161 & 0.5 \\
\hline 11 & 0.666778 & 0.55 \\
\hline 12 & 0.727394 & 0.6 \\
\hline 13 & 0.78801 & 0.65 \\
\hline 14 & 0.848626 & 0.7 \\
\hline 15 & 0.909242 & 0.75 \\
\hline 16 & 0.969858 & 0.8 \\
\hline 17 & 1.030474 & 0.85 \\
\hline 18 & 1.091091 & 0.9 \\
\hline 19 & 1.151707 & 0.95 \\
\hline 20 & 1.212323 & 1 \\
\hline 21 & 1.272939 & 1.05 \\
\hline 22 & 1.333555 & 1.1 \\
\hline 23 & 1.394171 & 1.15 \\
\hline 24 & 1.454788 & 1.2 \\
\hline 25 & 1.515404 & 1.25 \\
\hline 26 & 1.57602 & 1.3 \\
\hline 27 & 1.636636 & 1.35 \\
\hline 28 & 1.697252 & 1.4 \\
\hline 29 & 1.757868 & 1.45 \\
\hline 30 & 1.818484 & 1.5 \\
\hline
\end{tabular}




\begin{tabular}{|c|c|c|}
\hline 31 & 1.879101 & 1.55 \\
\hline 32 & 1.939717 & 1.6 \\
\hline 33 & 2.000333 & 1.65 \\
\hline 34 & 2.060949 & 1.7 \\
\hline 35 & 2.121565 & 1.75 \\
\hline 36 & 2.182181 & 1.8 \\
\hline 37 & 2.242797 & 1.85 \\
\hline 38 & 2.303414 & 1.9 \\
\hline 39 & 2.36403 & 1.95 \\
\hline 40 & 2.424646 & 2 \\
\hline 41 & 2.485262 & 2.05 \\
\hline 42 & 2.545878 & 2.1 \\
\hline 43 & 2.606494 & 2.15 \\
\hline 44 & 2.66711 & 2.2 \\
\hline 45 & 2.727727 & 2.25 \\
\hline 46 & 2.788343 & 2.3 \\
\hline 47 & 2.848959 & 2.35 \\
\hline 48 & 2.909575 & 2.4 \\
\hline 49 & 2.970191 & 2.45 \\
\hline 50 & 3.030807 & 2.5 \\
\hline 51 & 3.091423 & 2.55 \\
\hline 52 & 3.15204 & 2.6 \\
\hline 53 & 3.212656 & 2.65 \\
\hline 54 & 3.273272 & 2.7 \\
\hline 55 & 3.333888 & 2.75 \\
\hline 56 & 3.394504 & 2.8 \\
\hline 57 & 3.45512 & 2.85 \\
\hline 58 & 3.515737 & 2.9 \\
\hline 59 & 3.576353 & 2.95 \\
\hline 60 & 3.636969 & 3 \\
\hline 61 & 3.697585 & 3.05 \\
\hline 62 & 3.758201 & 3.1 \\
\hline 63 & 3.818817 & 3.15 \\
\hline 64 & 3.879433 & 3.2 \\
\hline 65 & 3.94005 & 3.25 \\
\hline 66 & 4.000666 & 3.3 \\
\hline 67 & 4.061282 & 3.35 \\
\hline 68 & 4.121898 & 3.4 \\
\hline 69 & 4.182514 & 3.45 \\
\hline 70 & 4.24313 & 3.5 \\
\hline 71 & 4.303746 & 3.55 \\
\hline 72 & 4.364363 & 3.6 \\
\hline 73 & 4.424979 & 3.65 \\
\hline 74 & 4.485595 & 3.7 \\
\hline 75 & 4.546211 & 3.75 \\
\hline 76 & 4.606827 & 3.8 \\
\hline 77 & 4.667443 & 3.85 \\
\hline
\end{tabular}




\begin{tabular}{|l|c|c|}
\hline 78 & 4.728059 & 3.9 \\
\hline 79 & 4.788676 & 3.95 \\
\hline 80 & 4.849292 & 4.05 \\
\hline 81 & 4.909908 & 4.1 \\
\hline 82 & 4.970524 & 4.15 \\
\hline 83 & 5.03114 & 4.2 \\
\hline 84 & 5.091756 & 4.25 \\
\hline 85 & 5.152372 & 4.3 \\
\hline 89 & 5.212989 & 4.35 \\
\hline 90 & 5.273605 & 4.4 \\
\hline
\end{tabular}

Table 4: Predictive and Experimental Value of Split Tensile Techniques at Different Curing Age.

\begin{tabular}{|c|c|c|}
\hline Curing Age & $\begin{array}{l}\text { Predictive Tensile Strength Variation of [W/C of } \\
\text { 0.46] }\end{array}$ & $\begin{array}{l}\text { Experimental Tensile Strength Variation of } \\
\text { [W/C of } 0.46]\end{array}$ \\
\hline 7 & 0.407675 & 0.378 \\
\hline 8 & 0.465915 & 0.432 \\
\hline 9 & 0.524154 & 0.486 \\
\hline 10 & 0.582394 & 0.54 \\
\hline 11 & 0.640633 & 0.594 \\
\hline 12 & 0.698872 & 0.648 \\
\hline 13 & 0.757112 & 0.702 \\
\hline 14 & 0.815351 & 0.756 \\
\hline 15 & 0.87359 & 0.81 \\
\hline 16 & 0.93183 & 0.864 \\
\hline 17 & 0.990069 & 0.918 \\
\hline 18 & 1.048308 & 0.972 \\
\hline 19 & 1.106548 & 1.026 \\
\hline 20 & 1.164787 & 1.08 \\
\hline 21 & 1.223026 & 1.134 \\
\hline 22 & 1.281266 & 1.188 \\
\hline 23 & 1.339505 & 1.242 \\
\hline 24 & 1.397744 & 1.296 \\
\hline 25 & 1.455984 & 1.35 \\
\hline 26 & 1.514223 & 1.404 \\
\hline 27 & 1.572463 & 1.458 \\
\hline 28 & 1.630702 & 1.512 \\
\hline 29 & 1.688941 & 1.566 \\
\hline 30 & 1.747181 & 1.62 \\
\hline 31 & 1.80542 & 1.674 \\
\hline 32 & 1.863659 & 1.728 \\
\hline 33 & 1.921899 & 1.782 \\
\hline 34 & 1.980138 & 1.836 \\
\hline 35 & 2.038377 & 1.89 \\
\hline 36 & 2.096617 & 1.944 \\
\hline 37 & 2.154856 & 1.998 \\
\hline
\end{tabular}




\begin{tabular}{|c|c|c|}
\hline 38 & 2.213095 & 2.052 \\
\hline 39 & 2.271335 & 2.106 \\
\hline 40 & 2.329574 & 2.16 \\
\hline 41 & 2.387814 & 2.214 \\
\hline 42 & 2.446053 & 2.268 \\
\hline 43 & 2.504292 & 2.322 \\
\hline 44 & 2.562532 & 2.376 \\
\hline 45 & 2.620771 & 2.43 \\
\hline 46 & 2.67901 & 2.484 \\
\hline 47 & 2.73725 & 2.538 \\
\hline 48 & 2.795489 & 2.592 \\
\hline 49 & 2.853728 & 2.646 \\
\hline 50 & 2.911968 & 2.7 \\
\hline 51 & 2.970207 & 2.754 \\
\hline 52 & 3.028446 & 2.808 \\
\hline 53 & 3.086686 & 2.862 \\
\hline 54 & 3.144925 & 2.916 \\
\hline 55 & 3.203164 & 2.97 \\
\hline 56 & 3.261404 & 3.024 \\
\hline 57 & 3.319643 & 3.078 \\
\hline 58 & 3.377883 & 3.132 \\
\hline 59 & 3.436122 & 3.186 \\
\hline 60 & 3.494361 & 3.24 \\
\hline 61 & 3.552601 & 3.294 \\
\hline 62 & 3.61084 & 3.348 \\
\hline 63 & 3.669079 & 3.402 \\
\hline 64 & 3.727319 & 3.456 \\
\hline 65 & 3.785558 & 3.51 \\
\hline 66 & 3.843797 & 3.564 \\
\hline 67 & 3.902037 & 3.618 \\
\hline 68 & 3.960276 & 3.672 \\
\hline 69 & 4.018515 & 3.726 \\
\hline 70 & 4.076755 & 3.78 \\
\hline 71 & 4.134994 & 3.834 \\
\hline 72 & 4.193233 & 3.888 \\
\hline 73 & 4.251473 & 3.942 \\
\hline 74 & 4.309712 & 3.996 \\
\hline 75 & 4.367952 & 4.05 \\
\hline 76 & 4.426191 & 4.104 \\
\hline 77 & 4.48443 & 4.158 \\
\hline 78 & 4.54267 & 4.212 \\
\hline 79 & 4.600909 & 4.266 \\
\hline 80 & 4.659148 & 4.32 \\
\hline 81 & 4.717388 & 4.374 \\
\hline 82 & 4.775627 & 4.428 \\
\hline 83 & 4.833866 & 4.482 \\
\hline 84 & 4.892106 & 4.536 \\
\hline 85 & 4.950345 & 4.59 \\
\hline
\end{tabular}




\begin{tabular}{|l|l|r|}
\hline 86 & 5.008584 & 4.644 \\
\hline 87 & 5.066824 & 4.698 \\
\hline 88 & 5.125063 & 4.752 \\
\hline 89 & 5.183302 & 4.806 \\
\hline 90 & 5.241542 & 4.86 \\
\hline
\end{tabular}

Table 5: Predictive and Experimental Value of Split Tensile Techniques at Different Curing Age.

\begin{tabular}{|c|c|c|}
\hline Curing Age & $\begin{array}{l}\text { Predictive Tensile Strength Variation of }[\mathrm{W} / \mathrm{C} \text { of } \\
0.48]\end{array}$ & $\begin{array}{l}\text { Experimental Tensile Strength Variation of } \\
\text { [W/C of } 0.48]\end{array}$ \\
\hline 7 & 0.39169 & 0.371 \\
\hline 8 & 0.447646 & 0.424 \\
\hline 9 & 0.503602 & 0.477 \\
\hline 10 & 0.559558 & 0.53 \\
\hline 11 & 0.615513 & 0.583 \\
\hline 12 & 0.671469 & 0.636 \\
\hline 13 & 0.727425 & 0.689 \\
\hline 14 & 0.783381 & 0.742 \\
\hline 15 & 0.839336 & 0.795 \\
\hline 16 & 0.895292 & 0.848 \\
\hline 17 & 0.951248 & 0.901 \\
\hline 18 & 1.007204 & 0.954 \\
\hline 19 & 1.063159 & 1.007 \\
\hline 20 & 1.119115 & 1.06 \\
\hline 21 & 1.175071 & 1.113 \\
\hline 22 & 1.231027 & 1.166 \\
\hline 23 & 1.286982 & 1.219 \\
\hline 24 & 1.342938 & 1.272 \\
\hline 25 & 1.398894 & 1.325 \\
\hline 26 & 1.45485 & 1.378 \\
\hline 27 & 1.510805 & 1.431 \\
\hline 28 & 1.566761 & 1.484 \\
\hline 29 & 1.622717 & 1.537 \\
\hline 30 & 1.678673 & 1.59 \\
\hline 31 & 1.734628 & 1.643 \\
\hline 32 & 1.790584 & 1.696 \\
\hline 33 & 1.84654 & 1.749 \\
\hline 34 & 1.902496 & 1.802 \\
\hline 35 & 1.958451 & 1.855 \\
\hline 36 & 2.014407 & 1.908 \\
\hline 37 & 2.070363 & 1.961 \\
\hline 38 & 2.126319 & 2.014 \\
\hline 39 & 2.182274 & 2.067 \\
\hline 40 & 2.23823 & 2.12 \\
\hline 41 & 2.294186 & 2.173 \\
\hline 42 & 2.350142 & 2.226 \\
\hline 43 & 2.406098 & 2.279 \\
\hline 44 & 2.462053 & 2.332 \\
\hline 45 & 2.518009 & 2.385 \\
\hline
\end{tabular}




\begin{tabular}{|c|c|c|}
\hline 46 & 2.573965 & 2.438 \\
\hline 47 & 2.629921 & 2.491 \\
\hline 48 & 2.685876 & 2.544 \\
\hline 49 & 2.741832 & 2.597 \\
\hline 50 & 2.797788 & 2.65 \\
\hline 51 & 2.853744 & 2.703 \\
\hline 52 & 2.909699 & 2.756 \\
\hline 53 & 2.965655 & 2.809 \\
\hline 54 & 3.021611 & 2.862 \\
\hline 55 & 3.077567 & 2.915 \\
\hline 56 & 3.133522 & 2.968 \\
\hline 57 & 3.189478 & 3.021 \\
\hline 58 & 3.245434 & 3.074 \\
\hline 59 & 3.30139 & 3.127 \\
\hline 60 & 3.357345 & 3.18 \\
\hline 61 & 3.413301 & 3.233 \\
\hline 62 & 3.469257 & 3.286 \\
\hline 63 & 3.525213 & 3.339 \\
\hline 64 & 3.581168 & 3.392 \\
\hline 65 & 3.637124 & 3.445 \\
\hline 66 & 3.69308 & 3.498 \\
\hline 67 & 3.749036 & 3.551 \\
\hline 68 & 3.804991 & 3.604 \\
\hline 69 & 3.860947 & 3.657 \\
\hline 70 & 3.916903 & 3.71 \\
\hline 71 & 3.972859 & 3.763 \\
\hline 72 & 4.028814 & 3.816 \\
\hline 73 & 4.08477 & 3.869 \\
\hline 74 & 4.140726 & 3.922 \\
\hline 75 & 4.196682 & 3.975 \\
\hline 76 & 4.252637 & 4.028 \\
\hline 77 & 4.308593 & 4.081 \\
\hline 78 & 4.364549 & 4.134 \\
\hline 79 & 4.420505 & 4.187 \\
\hline 80 & 4.47646 & 4.24 \\
\hline 81 & 4.532416 & 4.293 \\
\hline 82 & 4.588372 & 4.346 \\
\hline 83 & 4.644328 & 4.399 \\
\hline 84 & 4.700284 & 4.452 \\
\hline 85 & 4.756239 & 4.505 \\
\hline 86 & 4.812195 & 4.558 \\
\hline 87 & 4.868151 & 4.611 \\
\hline 88 & 4.924107 & 4.664 \\
\hline 89 & 4.980062 & 4.717 \\
\hline 90 & 5.036018 & 4.77 \\
\hline
\end{tabular}


Table 6a: Variation of Predictive Values of Split Tensile Techniques at Different Water cement Ratios Curing Age.

\begin{tabular}{|c|c|c|c|c|c|}
\hline $\begin{array}{l}\text { Variation of }[\mathrm{W} / \mathrm{C}] \text { on } \\
\text { Tensile strength }\end{array}$ & 0.4 & 0.42 & 0.44 & 0.46 & 0.48 \\
\hline $7 \mathrm{fcu}$ & 0.459653 & 0.44163 & 0.424313 & 0.407675 & 0.39169 \\
\hline $8 \mathrm{fcu}$ & 0.525318 & 0.50472 & 0.484929 & 0.465915 & 0.447646 \\
\hline $9 \mathrm{fcu}$ & 0.590982 & 0.567809 & 0.545545 & 0.524154 & 0.503602 \\
\hline $10 \mathrm{fcu}$ & 0.656647 & 0.630899 & 0.606161 & 0.582394 & 0.559558 \\
\hline $11 \mathrm{fcu}$ & 0.722312 & 0.693989 & 0.666778 & 0.640633 & 0.615513 \\
\hline $12 \mathrm{fcu}$ & 0.787976 & 0.757079 & 0.727394 & 0.698872 & 0.671469 \\
\hline $13 f c u$ & 0.853641 & 0.820169 & 0.78801 & 0.757112 & 0.727425 \\
\hline 14 fcu & 0.919306 & 0.883259 & 0.848626 & 0.815351 & 0.783381 \\
\hline $15 \mathrm{fcu}$ & 0.98497 & 0.946349 & 0.909242 & 0.87359 & 0.839336 \\
\hline $16 \mathrm{fcu}$ & 1.050635 & 1.009439 & 0.969858 & 0.93183 & 0.895292 \\
\hline $17 \mathrm{fcu}$ & 1.1163 & 1.072529 & 1.030474 & 0.990069 & 0.951248 \\
\hline $18 \mathrm{fcu}$ & 1.181964 & 1.135619 & 1.091091 & 1.048308 & 1.007204 \\
\hline $19 \mathrm{fcu}$ & 1.247629 & 1.198709 & 1.151707 & 1.106548 & 1.063159 \\
\hline $20 \mathrm{fcu}$ & 1.313294 & 1.261799 & 1.212323 & 1.164787 & 1.119115 \\
\hline $21 \mathrm{fcu}$ & 1.378958 & 1.324889 & 1.272939 & 1.223026 & 1.175071 \\
\hline $22 \mathrm{fcu}$ & 1.444623 & 1.387979 & 1.333555 & 1.281266 & 1.231027 \\
\hline $23 \mathrm{fcu}$ & 1.510288 & 1.451069 & 1.394171 & 1.339505 & 1.286982 \\
\hline $24 \mathrm{fcu}$ & 1.575953 & 1.514159 & 1.454788 & 1.397744 & 1.342938 \\
\hline $25 f c u$ & 1.641617 & 1.577248 & 1.515404 & 1.455984 & 1.398894 \\
\hline $26 \mathrm{fcu}$ & 1.707282 & 1.640338 & 1.57602 & 1.514223 & 1.45485 \\
\hline $27 f c u$ & 1.772947 & 1.703428 & 1.636636 & 1.572463 & 1.510805 \\
\hline $28 \mathrm{fcu}$ & 1.838611 & 1.766518 & 1.697252 & 1.630702 & 1.566761 \\
\hline $29 \mathrm{fcu}$ & 1.904276 & 1.829608 & 1.757868 & 1.688941 & 1.622717 \\
\hline $30 \mathrm{fcu}$ & 1.969941 & 1.892698 & 1.818484 & 1.747181 & 1.678673 \\
\hline
\end{tabular}

Table 6b: Variation of Predictive Values of Split Tensile Techniques at Different Water cement Ratios Curing Age.

\begin{tabular}{|c|c|c|c|c|c|}
\hline $\begin{array}{l}\text { Variation of }[\mathrm{W} / \mathrm{C}] \text { on } \\
\text { Tensile strength }\end{array}$ & 0.4 & 0.42 & 0.44 & 0.46 & 0.48 \\
\hline $31 \mathrm{fcu}$ & 2.035605 & 1.955788 & 1.879101 & 1.80542 & 1.734628 \\
\hline $32 \mathrm{fcu}$ & 2.10127 & 2.018878 & 1.939717 & 1.863659 & 1.790584 \\
\hline $33 \mathrm{fcu}$ & 2.166935 & 2.081968 & 2.000333 & 1.921899 & 1.84654 \\
\hline $34 \mathrm{fcu}$ & 2.232599 & 2.145058 & 2.060949 & 1.980138 & 1.902496 \\
\hline $35 \mathrm{fcu}$ & 2.298264 & 2.208148 & 2.121565 & 2.038377 & 1.958451 \\
\hline $36 \mathrm{fcu}$ & 2.363929 & 2.271238 & 2.182181 & 2.096617 & 2.014407 \\
\hline $37 \mathrm{fcu}$ & 2.429593 & 2.334328 & 2.242797 & 2.154856 & 2.070363 \\
\hline $38 \mathrm{fcu}$ & 2.495258 & 2.397418 & 2.303414 & 2.213095 & 2.126319 \\
\hline $39 \mathrm{fcu}$ & 2.560923 & 2.460508 & 2.36403 & 2.271335 & 2.182274 \\
\hline $40 \mathrm{fcu}$ & 2.626588 & 2.523598 & 2.424646 & 2.329574 & 2.23823 \\
\hline $41 \mathrm{fcu}$ & 2.692252 & 2.586687 & 2.485262 & 2.387814 & 2.294186 \\
\hline $42 \mathrm{fcu}$ & 2.757917 & 2.649777 & 2.545878 & 2.446053 & 2.350142 \\
\hline $43 \mathrm{fcu}$ & 2.823582 & 2.712867 & 2.606494 & 2.504292 & 2.406098 \\
\hline $44 \mathrm{fcu}$ & 2.889246 & 2.775957 & 2.66711 & 2.562532 & 2.462053 \\
\hline $45 \mathrm{fcu}$ & 2.954911 & 2.839047 & 2.727727 & 2.620771 & 2.518009 \\
\hline $46 \mathrm{fcu}$ & 3.020576 & 2.902137 & 2.788343 & 2.67901 & 2.573965 \\
\hline $47 f c u$ & 3.08624 & 2.965227 & 2.848959 & 2.73725 & 2.629921 \\
\hline
\end{tabular}




\begin{tabular}{|c|c|c|c|c|c|}
\hline $48 \mathrm{fcu}$ & 3.151905 & 3.028317 & 2.909575 & 2.795489 & 2.685876 \\
\hline $49 \mathrm{fcu}$ & 3.21757 & 3.091407 & 2.970191 & 2.853728 & 2.741832 \\
\hline $50 \mathrm{fcu}$ & 3.283234 & 3.154497 & 3.030807 & 2.911968 & 2.797788 \\
\hline $51 \mathrm{fcu}$ & 3.348899 & 3.217587 & 3.091423 & 2.970207 & 2.853744 \\
\hline $52 \mathrm{fcu}$ & 3.414564 & 3.280677 & 3.15204 & 3.028446 & 2.909699 \\
\hline $53 \mathrm{fcu}$ & 3.480228 & 3.343767 & 3.212656 & 3.086686 & 2.965655 \\
\hline $54 \mathrm{fcu}$ & 3.545893 & 3.406857 & 3.273272 & 3.144925 & 3.021611 \\
\hline $55 \mathrm{fcu}$ & 3.611558 & 3.469947 & 3.333888 & 3.203164 & 3.077567 \\
\hline $56 \mathrm{fcu}$ & 3.677223 & 3.533037 & 3.394504 & 3.261404 & 3.133522 \\
\hline $57 \mathrm{fcu}$ & 3.742887 & 3.596127 & 3.45512 & 3.319643 & 3.189478 \\
\hline $58 \mathrm{fcu}$ & 3.808552 & 3.659216 & 3.515737 & 3.377883 & 3.245434 \\
\hline $59 \mathrm{fcu}$ & 3.874217 & 3.722306 & 3.576353 & 3.436122 & 3.30139 \\
\hline $60 \mathrm{fcu}$ & 3.939881 & 3.785396 & 3.636969 & 3.494361 & 3.357345 \\
\hline
\end{tabular}

Table 6C: Variation of Predictive Values of Split Tensile Techniques at Different Water cement Ratios Curing Age.

\begin{tabular}{|c|c|c|c|c|c|}
\hline $\begin{array}{c}\text { Predictive Variation } \\
\text { of }[W / C] \text { on Tensile } \\
\text { strength }\end{array}$ & 0.4 & 0.42 & 0.44 & 0.46 & 0.48 \\
\hline $61 \mathrm{fcu}$ & 4.005546 & 3.848486 & 3.697585 & 3.552601 & 3.413301 \\
\hline $62 \mathrm{fcu}$ & 4.071211 & 3.911576 & 3.758201 & 3.61084 & 3.469257 \\
\hline $63 \mathrm{fcu}$ & 4.136875 & 3.974666 & 3.818817 & 3.669079 & 3.525213 \\
\hline $64 \mathrm{fcu}$ & 4.20254 & 4.037756 & 3.879433 & 3.727319 & 3.581168 \\
\hline $65 \mathrm{fcu}$ & 4.268205 & 4.100846 & 3.94005 & 3.785558 & 3.637124 \\
\hline $66 \mathrm{fcu}$ & 4.333869 & 4.163936 & 4.000666 & 3.843797 & 3.69308 \\
\hline $67 \mathrm{fcu}$ & 4.399534 & 4.227026 & 4.061282 & 3.902037 & 3.749036 \\
\hline $68 \mathrm{fcu}$ & 4.465199 & 4.290116 & 4.121898 & 3.960276 & 3.804991 \\
\hline $69 \mathrm{fcu}$ & 4.530863 & 4.353206 & 4.182514 & 4.018515 & 3.860947 \\
\hline $70 \mathrm{fcu}$ & 4.596528 & 4.416296 & 4.24313 & 4.076755 & 3.916903 \\
\hline $71 \mathrm{fcu}$ & 4.662193 & 4.479386 & 4.303746 & 4.134994 & 3.972859 \\
\hline $72 \mathrm{fcu}$ & 4.727858 & 4.542476 & 4.364363 & 4.193233 & 4.028814 \\
\hline $73 \mathrm{fcu}$ & 4.793522 & 4.605566 & 4.424979 & 4.251473 & 4.08477 \\
\hline $74 \mathrm{fcu}$ & 4.859187 & 4.668655 & 4.485595 & 4.309712 & 4.140726 \\
\hline $75 \mathrm{fcu}$ & 4.924852 & 4.731745 & 4.546211 & 4.367952 & 4.196682 \\
\hline $76 \mathrm{fcu}$ & 4.990516 & 4.794835 & 4.606827 & 4.426191 & 4.252637 \\
\hline $77 \mathrm{fcu}$ & 5.056181 & 4.857925 & 4.667443 & 4.48443 & 4.308593 \\
\hline $78 \mathrm{fcu}$ & 5.121846 & 4.921015 & 4.728059 & 4.54267 & 4.364549 \\
\hline $79 \mathrm{fcu}$ & 5.18751 & 4.984105 & 4.788676 & 4.600909 & 4.420505 \\
\hline $80 \mathrm{fcu}$ & 5.253175 & 5.047195 & 4.849292 & 4.659148 & 4.47646 \\
\hline $81 \mathrm{fcu}$ & 5.31884 & 5.110285 & 4.909908 & 4.717388 & 4.532416 \\
\hline $82 \mathrm{fcu}$ & 5.384504 & 5.173375 & 4.970524 & 4.775627 & 4.588372 \\
\hline $83 \mathrm{fcu}$ & 5.450169 & 5.236465 & 5.03114 & 4.833866 & 4.644328 \\
\hline $84 \mathrm{fcu}$ & 5.515834 & 5.299555 & 5.091756 & 4.892106 & 4.700284 \\
\hline $85 f c u$ & 5.581498 & 5.362645 & 5.152372 & 4.950345 & 4.756239 \\
\hline $86 f c u$ & 5.647163 & 5.425735 & 5.212989 & 5.008584 & 4.812195 \\
\hline $87 \mathrm{fcu}$ & 5.712828 & 5.488825 & 5.273605 & 5.066824 & 4.868151 \\
\hline $88 \mathrm{fcu}$ & 5.778493 & 5.551915 & 5.334221 & 5.125063 & 4.924107 \\
\hline $89 \mathrm{fcu}$ & 5.844157 & 5.615005 & 5.394837 & 5.183302 & 4.980062 \\
\hline $90 \mathrm{fcu}$ & 5.909822 & 5.678094 & 5.455453 & 5.241542 & 5.036018 \\
\hline
\end{tabular}




\section{Conclusion}

The aggregate size were observed in this study to monitor the rate of effect it has on the tensile strength of concrete, this was applied using Metakaolin as partial replacement of cement, aggregate sized applied on the study were heterogeneous in size, the results were generated from applied modeling and simulation through analytical solutions, the results generated linear trend were the optimum values were observed at ninety days of curing, the results at various mixed design experienced heterogeneity in tensile strength, this condition were based on the reflection of concrete property from aggregate variations in size, the compaction and this type of curing application based these condition including environmental factors. The simulation considered these parameters in the system to ensure that the influential reflections of these parameters are observed in the system. The study has expressed the variation impact on the aggregate heterogeneity based on the water cement ratios, the study observed the trend experiencing decrease in tensile as the water cement ratios increase, this implies that the behaviour of aggregate heterogeneity reflection stand out on its own, these are based on its major impact on the bond that is established on concrete materials as a property. The simulation monitored the behaviour of other concrete properties, but established precise reflection of impacts from the variation of aggregate size in the designed model concrete, the study is imperative because the rate of aggregate heterogeneity has been determined on various water cement ratios reflection through aggregate heterogeneous state in model concrete.

\section{References}

1. Topcu IB (1995) The Properties of Rubberized Concrete. Cement and Concrete Research 25(2): 304- 310.

2. Toutanji HA (1996) The use of Rubber Tyre Particles in Concrete to Replace Mineral Aggregate. Cement and Concrete Composites 18(2): 135-139.

3. Khatib ZR, Bayomy FM (1999) Rubberized Portland Cement Concrete. ASCE Journal of Materials in Civil Engineering 11(3): 206-213.

4. Ling TC (2011) Prediction of Density and Compressive Strength for Rubberized Concrete Block. Construction and Building Materials 25: 4303-4306.

5. Ohemeng EA, Yalley PPK (2013) Models for Predicting the Density and Compressive Strength of Rubberized Concrete Pavement Blocks. Construction and Building Materials 47: 656-661.

6. Eric AO, Peter PY, John D \& Susan Dzifa D (2014) Utilization of Waste Low Density Polyethylene in High Strengths Concrete Pavement Blocks Production. Civil and Environmental Research 6(5).
7. Ode T, Eluozo SN (2016) Predictive Model on Compressive Strength of Concrete Made with Locally 3/8 Gravel from Different Water Cement Ratios and Curing Age. International Journal of Scientific and Engineering Research 7(1): 1528-1551.

8. Choi YW, Moon DJ, Chung JS, Cho SK (2005) Effects of Waste PET Bottles Aggregate on Properties of Concrete. Cement and Concrete Research 35(4): 776-781.

9. Marzouk OY, Dheilly RM, Queneudec M (2007) Volarisation of PostConsumer Waste in Cementitious Concrete Composites. Waste Manag 27(2): 310-318.

10. Suganthy P, Chandrasekar D, Kumar SPK (2013) Utilization of Pulverized Plastic in Cement Concrete as Fine Aggregate. International Journal of Research in Engineering and Technology 2(6): 1015-1019.

11. Ode T, Eluozo SN (2016) Model Prediction to Monitor the Rate of Water Absorption of Concrete Pressured by Variation of Time and Water Cement Ratios. International Journal of Scientific and Engineering Research 7(1): 1514-1527.

12. Batayneh M, Marie I, Asi I (2007) Use of Selected Waste Materials in Concrete Mixes. Waste Management 27(12): 1870-1876.

13. Ode T, Eluozo SN (2016) Calibrating the Density of Concrete from Washed and Unwashed Locally 3/8 Gravel Material at Various Curing Age. International Journal of Scientific and Engineering Research $7(1): 1514-1552-15574$

14. Ode T, Eluozo SN (2016) Compressive Strength Calibration of Washed and Unwashed Locally Occurring 3/8 Gravel from Various Water Cement Ratios and Curing Age. International Journal Engineering and General Science 4(1): 462-483.

15. Naik TR, Singh SS, Huber CO, Brodersen BS (1996) Use of Post-Consumer Waste Plastics in Cement-Based Composites. Cement and Concrete Research 26:1489-1492.

16. Ode T, Eluozo SN (2016) Predictive Model to Monitor Variation of Concrete Density Influenced by Various Grade from Locally 3/8 Gravel at Different Curing Time. International Journal Engineering and General Science 4(1):502-522.

17. Ode T, Eluozo SN (2016) Predictive Model to Monitor Vitiation of StressStrain Relationship of 3/8 Gravel Concrete with Water Cement Ration [0.45] at Different Load. International Journal Engineering and General Science 4(1): 409-418.

18. Ode T (2004) Structural properties of Concrete made with locally occurring 3/8 gravel. M.TECH. Thesis Rivers State University of Science and Technology.

19. Ode T, Eluozo SN (2015) Predictive Model to Monitor the Variation of Concrete Density Influenced by various Grades from Locally 3/8 gravel at different curing time. International Journal of Engineering Research and General Science.

20. Chaudhary AS, Khan N, Kumar VP (2018) Analysis of Concrete Containing Different Admixtures as Partial Replacement of Cement. Int. Journal of Engineering Research and Application (Part -4) 7(7): 31-38. 


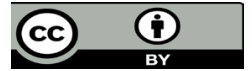

This work is licensed under Creative Commons Attribution 4.0 License

To Submit Your Article Click Here: Submit Article

DOI: $10.32474 /$ TCEIA.2020.04.000177

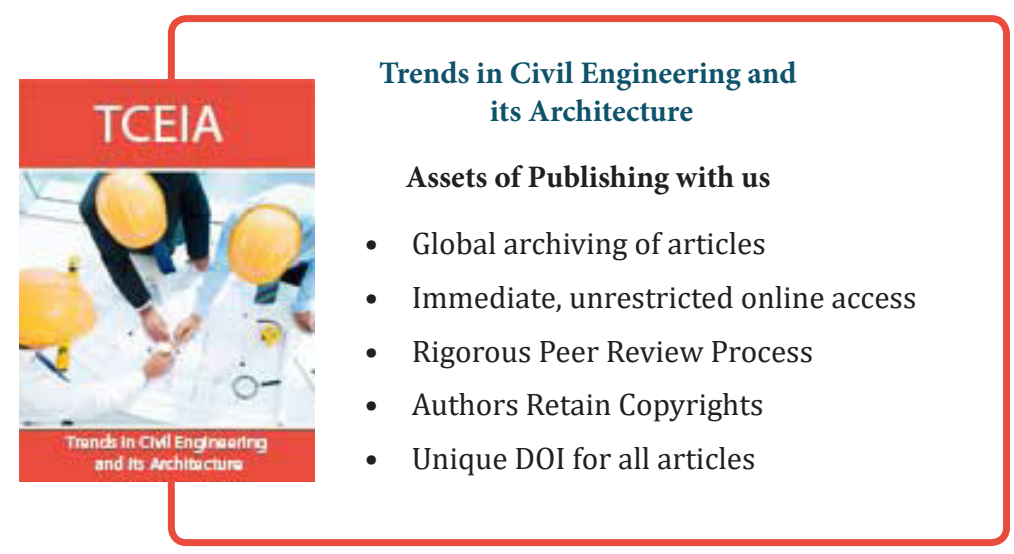

